\title{
Light harvesting by planar photonic crystal in solar cells: The case of amorphous silicon
}

\author{
Guillaume Gomard $^{1,2,3}$, Xianqin Meng ${ }^{1,2,3}$, Emmanuel Drouard ${ }^{1,2}$, Khalil El \\ Hajjam ${ }^{1,3}$, Emmanuel Gerelli ${ }^{1,3}$, Romain Peretti ${ }^{1,2}$, Alain Fave ${ }^{1,3}$, Regis \\ Orobtchouk $^{1,3}$, Mustapha Lemiti ${ }^{1,3}$ and Christian Seassal ${ }^{1,2}$ * \\ ${ }^{1}$ Université de Lyon, Institut des Nanotechnologies de Lyon (INL), UMR 5270, \\ CNRS-INSA-ECL-UCBL, France \\ ${ }^{2}$ Ecole Centrale de Lyon, 36 Avenue Guy de Collongue, 69134 Ecully Cedex, France \\ ${ }^{3}$ INSA de Lyon, Bat. Blaise Pascal, 7 Avenue Capelle, 69621 Villeurbanne, France
}

\begin{abstract}
In this paper, we discuss on light management in silicon thin film solar cells, using photonic crystals $(\mathrm{PhC})$ structures. We particularly focus on photovoltaic devices including amorphous silicon absorbers patterned as 2D PhCs. Physical principles and design rules leading to the optimized configuration of the patterned cell are discussed by means of optical simulations performed on realistic thin film solar cell stacks. Theoretically, a maximum $40 \%_{\text {rel }}$ increase of integrated absorption in the a-Si:H layer of the patterned cell is expected compared to the unpatterned case. Moreover, both simulation and optical characterization of the fabricated cells demonstrate the robustness of their optical properties with regards to the angle of incidence of the light and to the fabrication induced defects in the PhCs. Finally, the impact of the surface recombination due to the generation of new free surfaces with higher defect densities is addressed. We demonstrate that patterning still induces a substantial increase of the conversion efficiency, with a reasonable surface recombination velocity.
\end{abstract}

Keywords: Photonic crystals, Photovoltaic solar cell, Thin film solar cell, Hydrogenated amorphous silicon, Holographic lithography

\section{Introduction}

During the past decades, research in the field of photovoltaic (PV) solar cells was mainly driven by material development, technological upgrading and cost reduction. While bulk silicon solar cells are quite mature and widespread, the development of $2^{\text {nd }}$ generation devices, based on thin layers, tends to be limited. Actually, due to cost consideration and intrinsic defects in thin active PV layers, the efficiency is limited to values below $10 \%$ for single junction devices based on hydrogenated amorphous silicon (a-Si:H). New challenges have emerged with $3^{\text {rd }}$ generation PV devices, with a view to reach very high efficiency and low cost systems by using a wider spectral range. However, first attempts using non linear, quantum or other advanced effects which these new devices are based on, remain rather inefficient. In order to further improve the efficiency of thin film PV devices, or to increase the efficiency of the mechanisms related to $3^{\text {rd }}$ generation PV, the role of optics, including light trapping or photon harvesting, has recently appeared as a new and very promising route.

Within the past years, the relative maturity of nanophotonics has enabled the emergence of various new concepts for light management. At the same time, the development of low cost micro and nanotechnologies compatible with the patterning of wide areas has made the implementation of such

\footnotetext{
* Corresponding author: Christian.Seassal@ec-lyon.fr
} 
nanophotonic structures feasible. Among the widespread and promising approaches are those based on surface plasmons, including ordered top-down metallic grating and bottom-up integrated metallic nanoparticles [1], [2], [3]. Other routes make use of non metallic micro and nanostructures; this is typically the case of Photonic Crystal (PhC) structures, but also sub-wavelength structures (SWS) which may be patterned within the absorbing layer. The use of a $\mathrm{PhC}$ structure to control the absorption in a PV solar cell was proposed in 2006 [4]: High diffraction orders of a PhC back reflector were used to increase the photon path within the silicon solar cell. In 2008, we proposed to pattern a thin silicon layer as a $\mathrm{PhC}$, and to make profit of this structure to increase both incident light trapping, and photon lifetime in the absorbing layer [5]. The combination of such patterned absorbers with a top antireflection (AR) layer and back electrodes was then proposed for 1D and 2D PhCs [6], [7]. Both reports concluded an expected relative increase of the efficiency up to around $30 \%$, following to the integration of a $\mathrm{PhC}$ structure within the absorbing layer. As another approach to exploit $\mathrm{PhC}$ structures, the integration of conductive inverted opals as the intermediate layer of a tandem cell was proposed and investigated [8].

The case of hydrogenated amorphous silicon (a-Si:H) solar cells have attracted much attention, as a test vehicle for the implementation of various nanophotonic structures. Indeed, the thickness of such absorbing layers is of the order of the wavelength of incident light, which is comparable to the template used to fabricate nanophotonic structures for other applications as surface emitting light sources or optical filters. On this basis, various groups have implemented periodic structures like nanocones [9], metallic gratings [10], or SWS in solar cells [11]. The advantage of these approaches is that they remain very close to the standard technology used to fabricate the solar cells. However, these designs are little flexible, and the periodic perturbation remains relatively weak. An alternative method for providing light trapping in the cell consists in using a randomly textured substrate [12] on top of which are deposited the different layers. While this can lead to a broad absorption enhancement, it does not allow modal engineering to adjust the absorption on a specific wavelength range and more generally, is limited in its way of controlling absorption.

In this paper, we will focus on strongly corrugated $\mathrm{PhC}$ absorbers constituted of a-Si:H layers periodically patterned by nanolithography and etching. In section 2 , we will present the basic principles of light trapping and absorption control by a planar 2D PhC structure etched through an a$\mathrm{Si}: \mathrm{H}$ layer. The design and main properties of $\mathrm{PhC}$ a-Si:H based solar cells will be presented and discussed in section 3, including optical and electrical simulations, as well as various optical measurements.

\section{Properties of 2D photonic crystal absorbers}

As a basic structure to investigate the potential of $\mathrm{PhCs}$ for the control of sun light collection and absorption, we will consider a planar 2D PhC etched in an a-Si:H layer. A thickness of $100 \mathrm{~nm}$ is considered for this layer, which is lower than the mean free path of the minority carriers in this material. The main parameters of the $\mathrm{PhC}$ are then $L$, its lattice parameter and $f f$, the silicon surface filling fraction. In this paper, we will only consider square symmetry 2D PhC structures constituted by air holes drilled in the whole silicon layer thickness. ff is then given by $1-\pi r^{2} / L^{2}, r$ being the radius of the air holes.

The RCWA (Rigorous Coupled Wave Analysis) method [13] is well-suited to the simulation of the reflectance, transmittance and absorption spectra of such periodic structures, taking into account the dispersion characteristics of the considered media. This method is available in the CAMFRGARCLED [14] software package. For a-Si:H absorbers, the investigated spectral domain is limited from 300 to around $750 \mathrm{~nm}$. Indeed, the sun light intensity is negligible under the lower limit. Above approximately $750 \mathrm{~nm}$, close to band-gap of the a-Si:H, the extinction coefficient of the absorbing 
media falls below 0.001 , and the absorption is extremely weak. Regardless of electrical considerations, the efficiency of such an absorber may be calculated through the integration, over the whole spectral range mentioned above, and taking into account the $\mathrm{AM} 1.5 \mathrm{G}$ solar spectral intensity distribution. In this section, we will consider a PhC structure with $L=0.38 \mu \mathrm{m}$ and $D / L=62.5 \%$ (where $D=2 r$ ). These values will be justified in section 3 , as they correspond to an optimized set of parameters for a full solar cell stack. Figure 1 shows the absorption spectrum calculated for this isolated structure at normal incidence with a schematic view of the $2 \mathrm{D}$ planar $\mathrm{PhC}$ studied. The absorption spectrum of an unpatterned layer surrounded by air is also shown.

a)

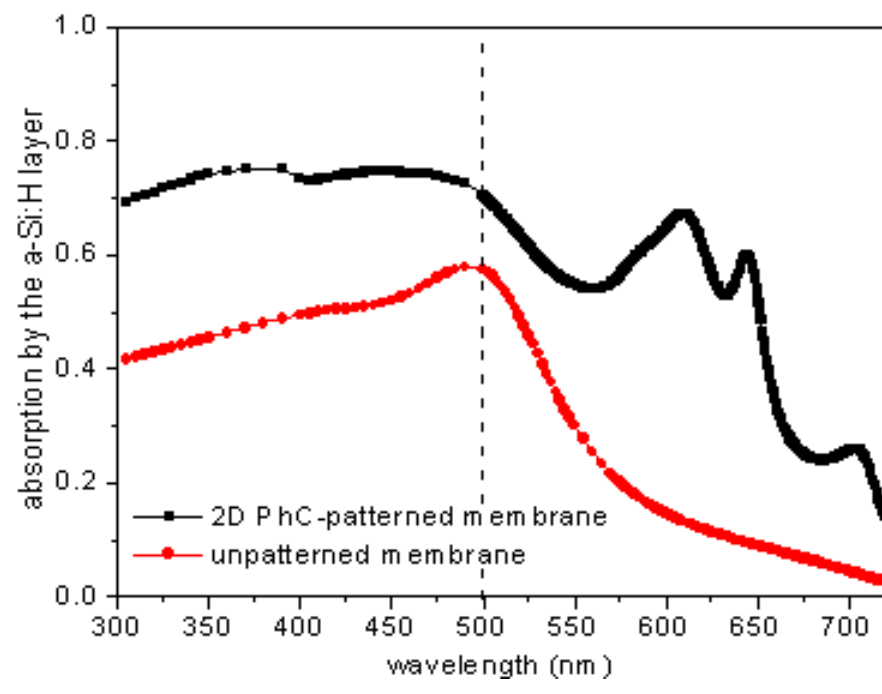

b)

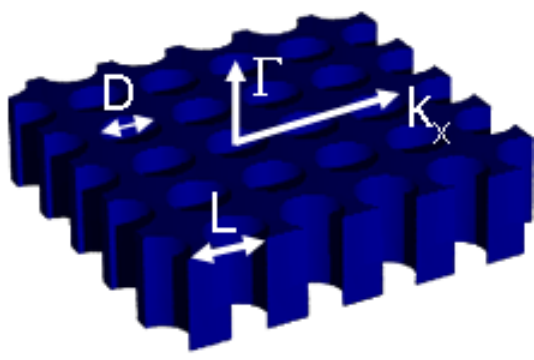

Figure 1. Simulated absorption spectrum of an optimized 2D PhC-patterned layer compared to its unpatterned equivalent (a) and schematic view of the planar 2D PhC with its characteristic parameters (b)

The spectrum corresponding to the PhC slab clearly exhibits two domains. At low wavelengths, due to the very high absorption coefficient of a-Si:H, light is immediately absorbed without any possibility to experience diffraction and interferences phenomena. Consequently, spectra appear flat up to about $500 \mathrm{~nm}$. Although the refractive index of the absorbing medium is fairly high, in excess of 4 , the reflectance is relatively weak thanks to the wavelength-scale pattern, which tends to act as an antireflective surface [11]. This explains the higher absorption than in the case of the non patterned slab. On the contrary, for wavelengths in excess of 500nm, series of broad and intense peaks clearly appear. They correspond to optical resonances, which are specific of the PhC structures, as they do not appear on the reference a-Si:H layer. Whatever the nature of the resonances, their impact on the absorption of sun light can be amplified if an optimized coupling between the incident optical wave and the absorbing medium is achieved. The unpatterned absorbing medium is characterized by its refractive index, $n$, and absorption coefficient, $\alpha$. One may introduce the term $\tau_{\alpha}$, the lifetime related to the absorption process in such a non patterned medium, which is related to the absorption coefficient, via $\alpha=\frac{1}{\tau_{\varangle}^{c} / n}$

resonance is $Q=\omega \tau_{0}$, where $\tau_{0}$ is the photon lifetime in the mode. On one hand, when $Q$ is decreased, both the coupling efficiency of incident light into the resonance and its spectral linewidth are increased. On the other hand, a higher $\tau_{0}$ enables higher light matter interaction between photons 
collected in the resonance and absorbing medium. Finally, as both incident light coupling and lightmatter interaction should be maximized, the adjustment of $Q$ results from a trade-off, namely the critical coupling conditions, which can be written in terms of lifetime: $\tau_{0}=\tau_{\varpi}$, or $Q=\frac{2 \pi n}{\alpha \lambda}$. Such a simple condition leads to a maximized 50\% absorption in a symmetric, single mode $\mathrm{PhC}$ membrane. In our case of interest, several modes are spectrally close enough to lead to an even larger absorption.

In order to optimize and control the behavior of our patterned absorbers, it is necessary to identify the nature of the resonances that appear in these spectra. To reach this goal, the band structure of the planar PhC was calculated using 3D Finite Difference Time Domain (FDTD) simulations. The isolated membrane was considered as transparent to facilitate the analysis and its refractive index was set to 4.25 , which corresponds to the value in the middle of the spectral range considered $(\lambda=650 \mathrm{~nm})$. These simplifications slightly modify the resonant frequency and quality factors of the modes studied but lead to the same conclusions as if one took the dispersion of the indices and absorption of a-Si:H into account. Periodic conditions are applied in $x$ and $y$ directions, whereas perfectly matched layers (PML) are placed on each side of the membrane, perpendicular to the $z$ axis. The excitation of the modes in the planar PhC is triggered by a current source located either on a high symmetry point in the middle of the unit cell (only for symmetric modes with regards to the $\mathrm{z}$ axis) or off-centered from such a particular point (for both antisymmetric + symmetric modes). The result of this simulation is shown in Figure 2.a. For the remainder of this section, we will consider a limited part of this band structure, corresponding to the wavelength range between 550 and $750 \mathrm{~nm}$, i.e. where the absorption coefficient of a-Si:H is low enough, so that incident light can really explore the $\mathrm{PhC}$ Bloch modes. Various modes, both symmetric and antisymmetric, appear around the $\Gamma$-point, i.e., corresponding to light incoming around the normal incidence. As the photonic bands appear quite flat around $\Gamma$, they correspond to slow light modes, which are in-plane resonances that can be excited by incident light. These modes are similar to those recently used to control laser emission or non linear optical phenomena, or to generate reflectors [15]. Absorption peaks can originate from the overlap between symmetric modes with the incoming plane wave, and consequently, the electromagnetic field is vertically concentrated in the middle of the layer or said differently, far from the doped regions when an a-Si:H PIN junction is considered. From the initial FDTD simulation, and using the filter diagonalization [16], we calculated the resonant frequency and quality factors of the Bloch modes, at normal $(\Gamma)$ and oblique incidence $\left(20^{\circ}\right)$, considering a transparent medium with a realistic refractive index. The results are shown in Figure 2.b, together with the absorption spectra calculated at 0 and $20^{\circ}$. A first observation is that most of the absorption peaks above $550 \mathrm{~nm}$ correspond to a symmetric Bloch mode of the PhC. It is therefore generally possible to correlate absorption spectra with slow light resonances, specifically at high wavelengths [7]. A second remark is that the integrated absorption is not significantly modified with the angle of incidence; it remains much higher than in the case of the unpatterned layer. However, considering specific wavelengths, if the absorption remains similar at 0 and $20^{\circ}$ for some of the modes, the behavior is more complex for a few absorption peaks. At $650 \mathrm{~nm}$, a Bloch mode with a relatively low Q-factor is only predicted at the $\Gamma$-point. Indeed, the corresponding absorption peak vanishes at $20^{\circ}$. Conversely, at $730 \mathrm{~nm}$, an absorption peak only appears for an angle of incidence of $20^{\circ}$. This last situation corresponds to a typical property of $2 \mathrm{D}$ $\mathrm{PhC}$ structures, where incoming light cannot be coupled at normal incidence, due to symmetry mismatch between the Bloch mode and the plane wave [17]. Finally, the high angular acceptance of these PhC absorbers, which was discussed in previous papers [18], [7], can be explained by different aspects. On the one hand, the low curvature of many of the photonic bands yields to a reasonable stability with regards to the angle of incidence. On the other hand, the complexity and the diversity of 
the Bloch modes which are involved in the absorption of sun light are such that while some vanish at oblique incidence, other appear and contribute to the global process.

On the basis of the concepts introduced above, a real and technologically feasible solar cell can be designed and investigated; this will be discussed in the following section.

a)
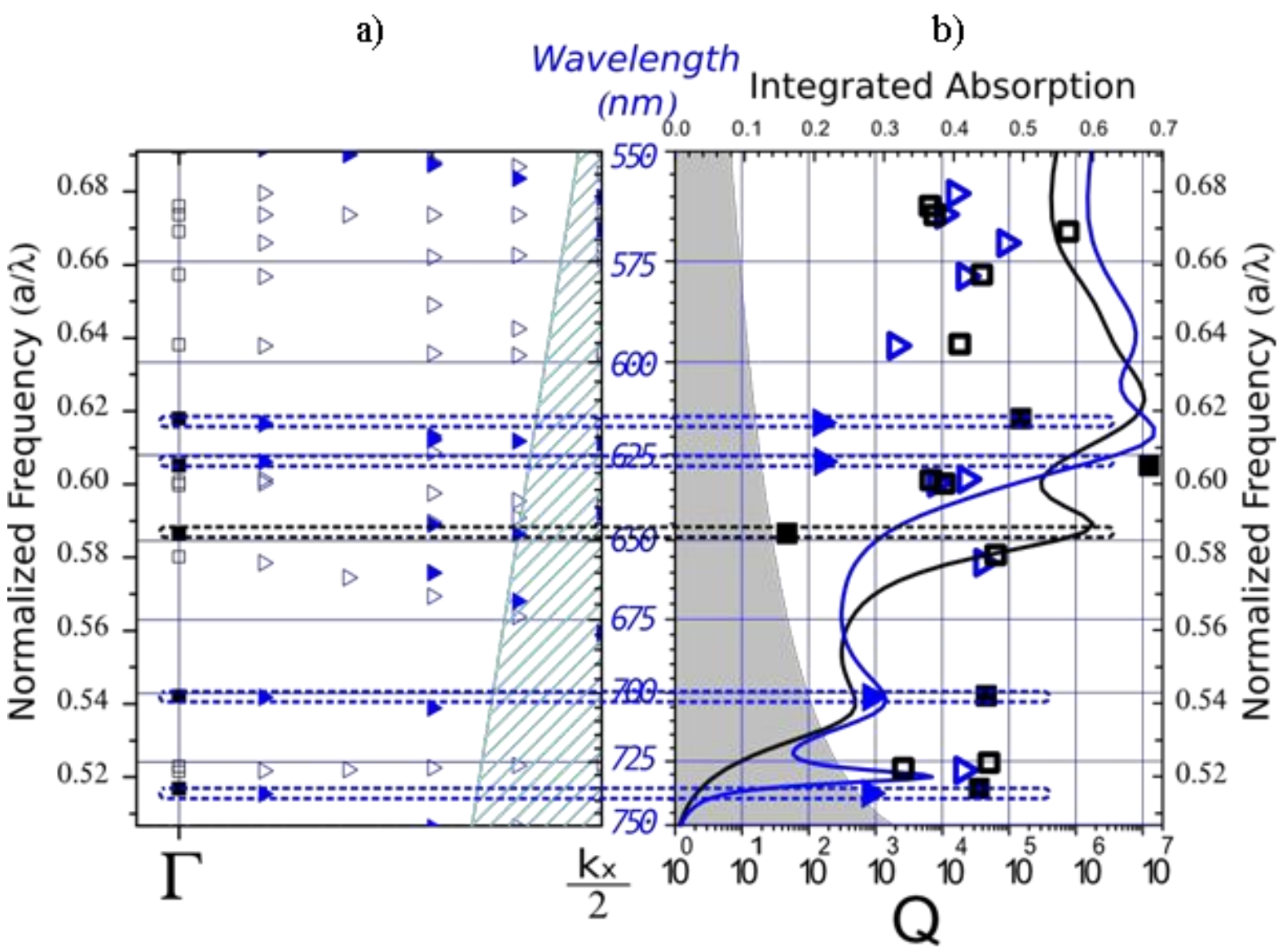

Figure 2. a) Band structure of a $2 \mathrm{D}$ PhC membrane $(L=0.38 \mu \mathrm{m}$ and $D / L=62.5 \%)$ made out of a $100 \mathrm{~nm}$ thick isolated a-Si:H layer $(n(\mathrm{a}-\mathrm{Si}: \mathrm{H})=4.25$ and $k(\mathrm{a}-\mathrm{Si}: \mathrm{H})=0)$. Dashed region corresponds to the domain beyond the $20^{\circ}$ light cone and $\mathrm{k}_{\mathrm{x}}$ is the nearest-neighbour direction as depicted on Figure 1. b)

Absorption spectra of this isolated 2D PhC membrane for a normal (black curve) and a $20^{\circ}$ (blue curve) angle of incidence. Symmetric (solid symbols) and anti-symmetric (hollow symbols) modes of this PhC membrane, which quality factors are denoted $Q$, are reported for the normal (squares) and the $20^{\circ}$ (triangles) angle of incidence. Upper limit of the grey region is associated with the value of $Q$ in the critical coupling condition $\left(Q_{c}=n(\mathrm{a}-\mathrm{Si}: \mathrm{H}) / 2 k(\mathrm{a}-\mathrm{Si}: \mathrm{H})\right)$

\section{Photonic crystal assisted solar cells}

\subsection{Optical design (and simulation)}

In order to take advantage of the unique properties of $\mathrm{PhCs}$, and so as to implement the basic structure introduced in section 2, a specific design is needed. Like in the case of several approaches based on the deposition of layers on a patterned substrate, the classical stack includes a metallic bottom contact, a PIN a-Si:H diode and a top transparent electrode made of a TCO (transparent and conductive oxide) 
like, e.g., ITO (Indium Tin oxide) [19]. However, in the case of a $\mathrm{PhC}$ structure, an important difference is that a high refractive index layer, for example the absorber, exhibits a periodic pattern, generally fabricated by etching, local deposition or growth, and with a lattice parameter of the order of the wavelength in the visible range. The typical design of a PhC solar cell structure based on a-Si:H is schematically displayed in Figure 3. The choice of ITO as the top layer is straightforward, since it enables the creation of a thin TCO with a conductivity which is sufficient to drive the electrical charges laterally, to metallic pads. Its thickness is limited to $50 \mathrm{~nm}$ in order to minimize the amount of light absorbed in this layer. In classical solar cells, the thickness of the core a-Si:H PIN stack is around 300 to $400 \mathrm{~nm}$. This value is chosen so as to reach a trade-off between light absorption and bulk carrier recombination. In the case of PhC solar cells, as the absorption is controlled and enhanced by resonances, the intrinsic a-Si:H layer may be reduced down to $100 \mathrm{~nm}$. This both results in lower carriers bulk recombination, and a strongly reduced deposition time of the stack. Both the top TCO layer and the a-Si:H PIN diode are periodically patterned by air holes. The bottom electrode is basically made of a thin unpatterned metallic layer, $\mathrm{Ag}$ being a relevant choice since it induces a limited light absorption. Such a layer acts at the same time as an optical mirror, which may control the dynamics of the optical resonances of the $\mathrm{PhC}$ structure.

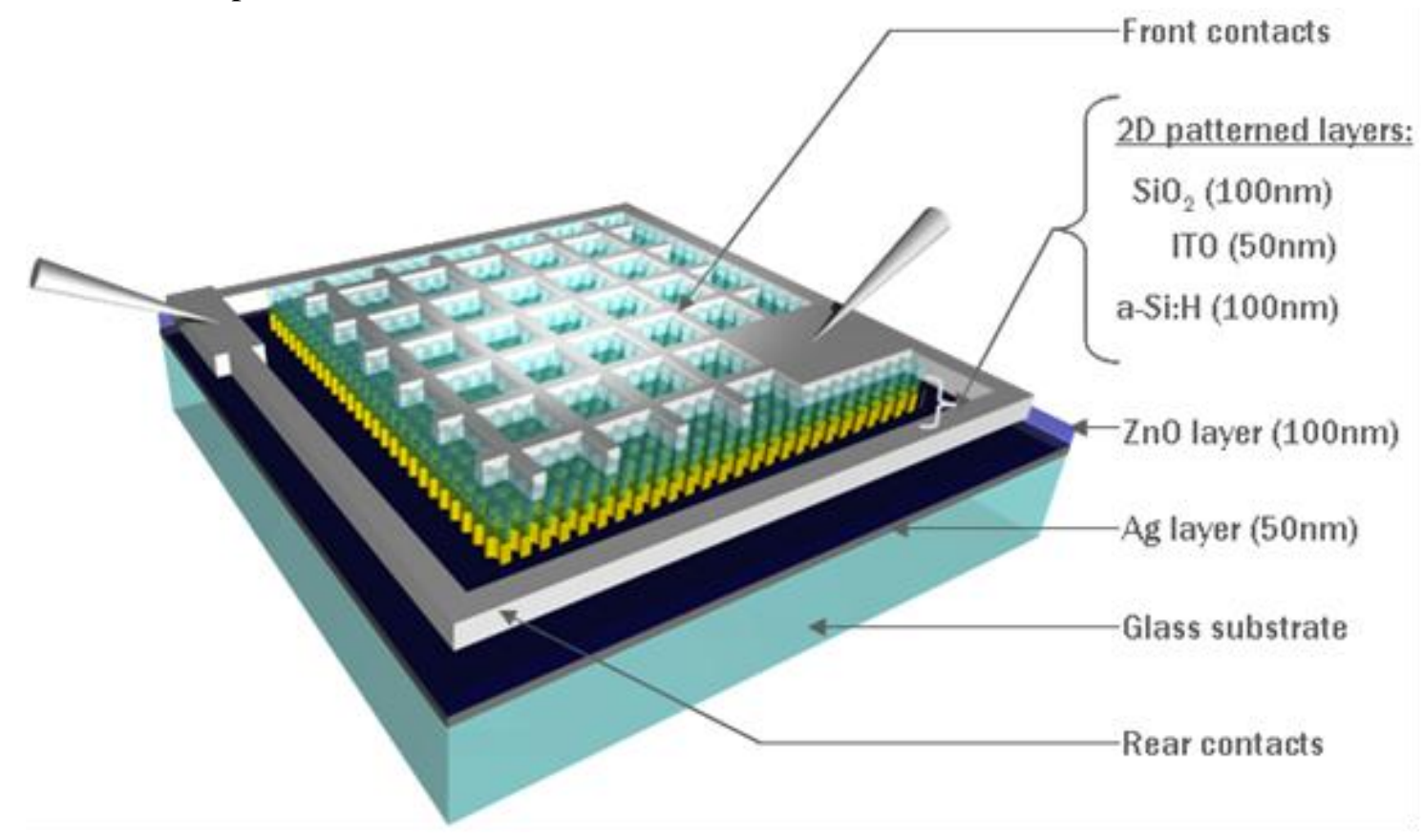

Figure 3. Schematic view of our 2D PhC-patterned cell on its glass substrate, with a top $\mathrm{SiO}_{2}$ layer added for technological purposes (not to scale). Tips represented on the figure will be used for contacting the front and rear metallic grids

The impact of such a reflector on the $Q$-factor of a $\mathrm{PhC}$ resonance was already demonstrated in previous papers, notably in the case of wide bandwidth reflectors or slow Bloch mode microlasers [20]. As in the case of these optical devices, the recycling of PhC Bloch modes is achieved by carefully adjusting the spacing between the mirror and the PhC structure. For this reason, but also because the presence of an oxide layer between $\mathrm{Ag}$ and a-Si:H is useful to limit material diffusion, a thin TCO spacer layer is generally introduced.

Scanning $L$ and the $D / L$ ratio over a technologically reasonable range, i.e., respectively between 300 and $700 \mathrm{~nm}$, and from 30 to $70 \%$, enables to determine the PhC structure which yields to the highest absorption efficiency over the whole spectral range considered. As in the case of section 2, the real 
dispersion characteristics of all the different materials is considered [7].Using this methodology, design parameters were obtained by maximizing the integrated absorption efficiency over the whole stack. Figure 4 exhibits the absorption spectra of solar cells with optimized geometrical parameters with or without a $\mathrm{ZnO}$ spacer layer, and with different thicknesses. In Table 1 are summarized the optimal geometrical parameters for three realistic values of the $\mathrm{ZnO}$ layer thickness, the integrated absorption efficiency over the whole wavelength range of interest, both in the whole stack and in the sole a-Si:H layer.

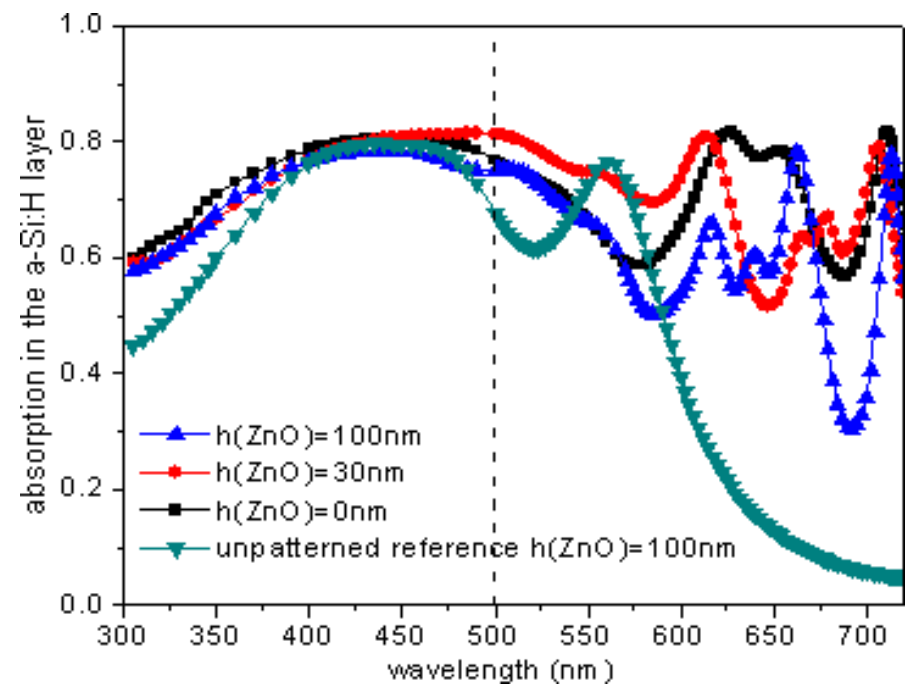

Figure 4. Simulated absorption spectra in the patterned a-Si:H layer of three optimized configurations with different $\mathrm{ZnO}$ layer thicknesses. Absorption spectrum of an unpatterned cell with $h(\mathrm{ZnO})=100 \mathrm{~nm}$ also reported for comparison

Table 1. Parameters and integrated absorptions (in \% of solar light intensity) for the optimized configurations using different $\mathrm{ZnO}$ layer thicknesses

\begin{tabular}{|c|c|c|c|}
\hline $\begin{array}{l}\text { ZnO layer thickness } \\
(\mathrm{nm})\end{array}$ & $\begin{array}{c}\text { 2D PhC } \\
\text { parameters }\end{array}$ & $\begin{array}{l}\text { Integrated absorption in } \\
\text { the whole stack }(\%)\end{array}$ & $\begin{array}{c}\text { Integrated absorption in } \\
\text { the a-Si:H layer }(\%)\end{array}$ \\
\hline 0 & $\begin{array}{c}L=0.34 \mu \mathrm{m}, \\
D / L=70 \%\end{array}$ & 87.8 & 72.4 \\
\hline 30 & $\begin{array}{c}L=0.44 \mu \mathrm{m}, \\
D / L=70 \%\end{array}$ & 88.3 & 72.5 \\
\hline 100 & $\begin{array}{l}L=0.38 \mu \mathrm{m}, \\
D / L=62.5 \%\end{array}$ & 82.0 & 65.7 \\
\hline
\end{tabular}

These integrated absorption values should be compared to the one obtained with an unpatterned stack, which is limited to $51.7 \%$ (in a-Si:H layer) in the case of the unpatterned stack with a $100 \mathrm{~nm} \mathrm{ZnO}$ thickness. It appears that considering both the efficiency and material diffusion issues, $\mathrm{ZnO}$ layer thickness can be limited to 30nm [21]. However, in the remainder of this section, only the optimized structure with a $100 \mathrm{~nm}$ thick $\mathrm{ZnO}$ layer will be considered, with the same $\mathrm{PhC}$ geometrical parameters as in the case of the structure discussed in section 2 . 
The robustness of the absorption with regards to technological imperfections in the PhC parameters $(L$ and the $D / L$ ratio) of the patterned cell was then evaluated. We first calculated the modification of the integrated absorption while the PhC parameters drift from the optimal configuration $(L=0.38 \mu \mathrm{m}$ and $D / L=62.5 \%$ in our case). As can be seen in Figure 5, a large domain characterized by a high absorption (close to 80\%) appears around this optimal configuration, so that a reasonable change in the $\mathrm{PhC}$ parameters should not strongly affect the optical properties of our patterned cell.

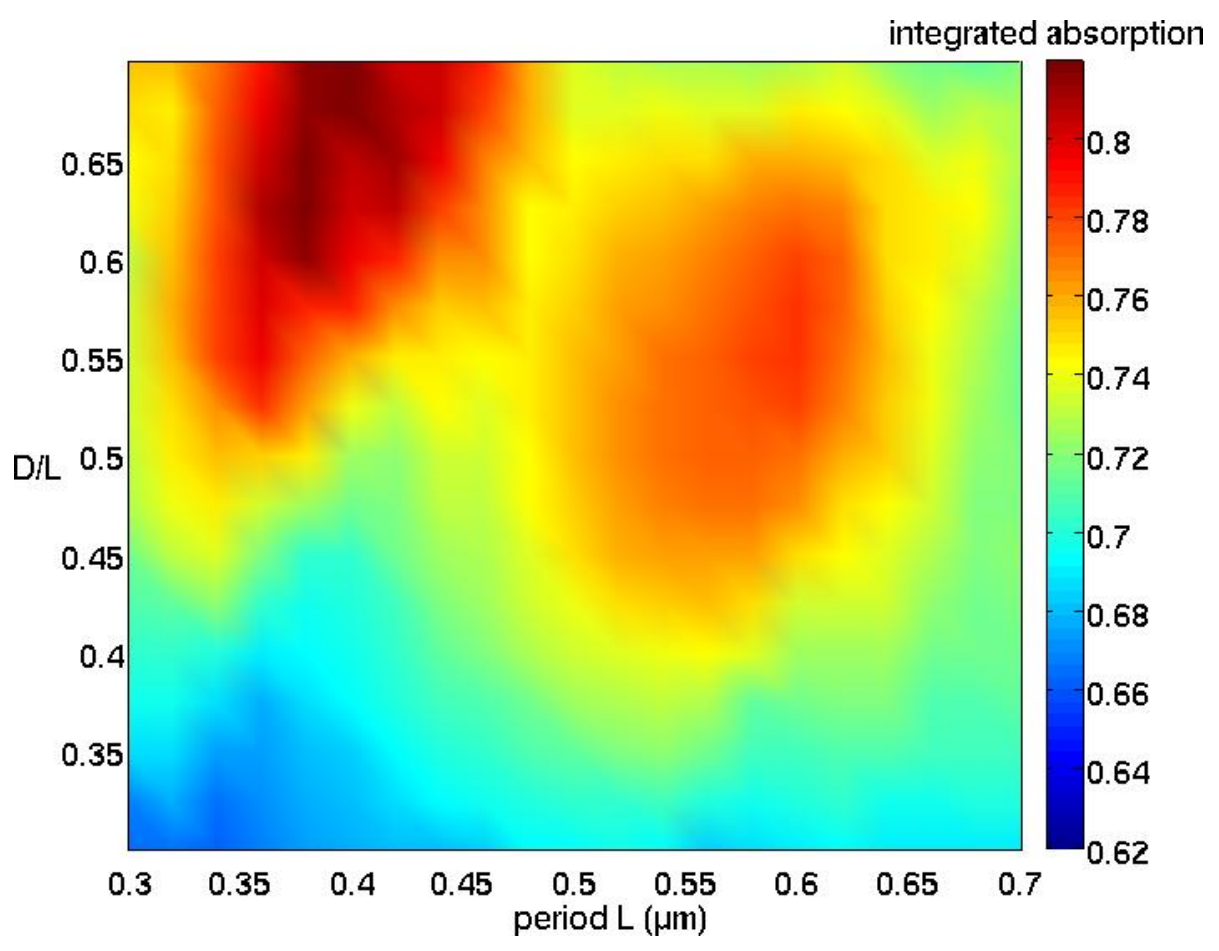

Figure 5. Contour map of the integrated absorption in the whole stack as a function of the period of the $\mathrm{PhC}$ and its $D / L$ ratio (RCWA simulations performed taking into account the AM1.5G spectrum)

Besides, we investigated the impact of inhomogeneities in the PhC parameters within a given cell. From the SEM images of different patterned samples, as those which will be further introduced in this paper, we observed that the period $L$ could be satisfactorily controlled during the patterning, whereas assuring a constant diameter of the holes over the whole surface of the cell was more demanding. Typically, a dispersion of $10 \%$ in size was measured for the latter parameter. A qualitative assessment of this effect on the absorption spectrum was achieved by FDTD simulations. A unit cell including 16 holes was then created in a 3D simulation window, by applying periodic conditions in both $x$ and $y$ directions and PML at the front and rear sides of the stacks. The absorption spectrum of the experimental configuration, which corresponds to the optimized one after randomly introducing $10 \%$ of dispersion in the hole diameter, is shown on Figure 6 together with the one of the perfect cell (i.e without dispersion). Since the spectral region between 550 and $720 \mathrm{~nm}$ is the most likely to be affected, only this wavelength range was simulated. 


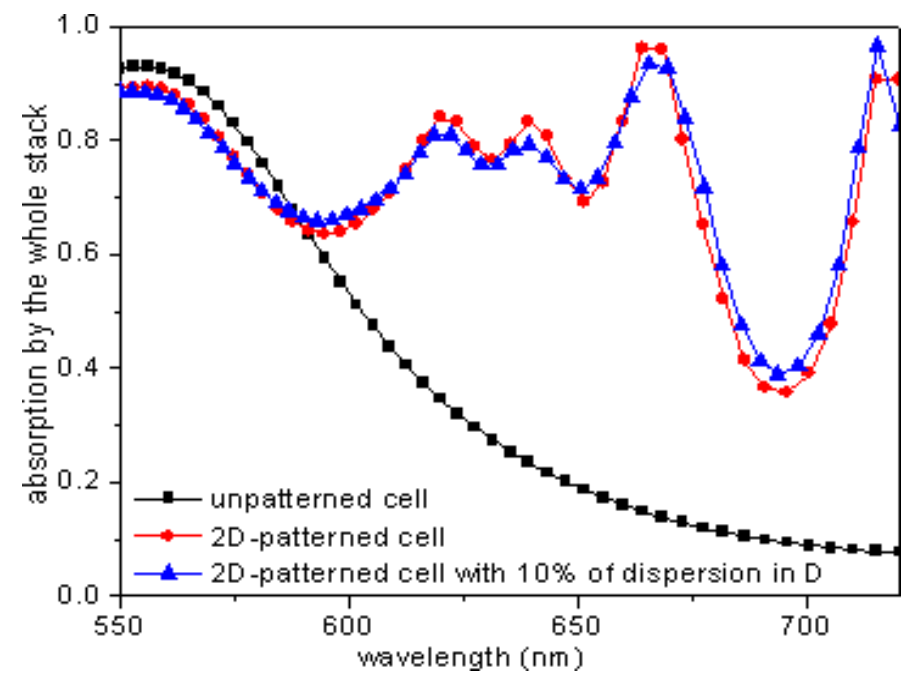

Figure 6. Effect of the hole diameter dispersion on the absorption spectrum of the optimized patterned cell $(h(\mathrm{ZnO}=100 \mathrm{~nm}))$. A unique period over the unit cell and a normal incidence of the light were considered

It then appears that this diameter inhomogeneity has almost no influence on the optical properties of the cell. Consequently, the optical design chosen for the fabrication, in addition to presenting a subsequent absorption gain, is fully compatible with the unavoidable technological imperfections introduced during the patterning steps.

\subsection{Fabrication}

The implementation of advanced photonic concepts should be achieved by limiting the additional manufacturing costs to really add value to the thin-film PV technology. Under this criterion, nanostamping and holographic lithography both appear as appropriate methods in order to process large area for the patterning of solar cells. In our case, we chose to develop a fabrication process using holographic lithography for its versatility and ease of implementation which enables to scan a large range of configurations.

As already discussed in the previous section, our $6 \times 6 \mathrm{~mm}^{2}$ cells are designed, from back to front, as follows: Glass(substrate) $/ \mathrm{Ag}_{50 \mathrm{~nm}} / \mathrm{ZnO}_{100 \mathrm{~nm}} / \mathrm{a}-\mathrm{Si}: \mathrm{H}(\mathrm{PIN})_{100 \mathrm{~nm}} / \mathrm{ITO}_{50 \mathrm{~nm}}$. An additional 100nm thick silica layer is deposited on the front TCO layer and will act as a hard mask during the etching steps performed by Reactive Ion Etching (RIE).

The first step consists in patterning a photosensitive resist on top of the hard mask as a square array of round holes. For that purpose, a 266nm laser is used for creating interference fringes in the photoresist. While the first exposure gives rise to a $1 \mathrm{D}$ pattern, shifting the sample by $90^{\circ}$ before the second one enables to obtain the targeted 2D pattern which geometrical parameters ( $L$ and $D$ ) are set by adjusting the impinging angle of the laser and the exposure time, among others. After development, a 2D template of holes is then formed and ready to be transferred into the underlying layers via RIE.

This is carried out by first patterning the hard mask by $\mathrm{CHF}_{3}$ at $15 \mathrm{mTorr}$, followed by a photoresist removal step by $\mathrm{O}_{2}$ plasma, and subsequently, keeping on the etching by $\mathrm{CH}_{4}: \mathrm{H}_{2}$ at 30 mTorr (ITO) and $\mathrm{SF}_{6}$ : Ar at $15 \mathrm{mTorr}(\mathrm{a}-\mathrm{Si}: \mathrm{H})$, until the $\mathrm{ZnO}$ layer is reached (See Figure 3). As it may be difficult to control the end-point of the etching, one can ensure the complete transfer of the pattern by slightly over-etching into this latter layer, since a reasonable change of its thickness does not significantly degrade the optical performance of the cell, as seen in section 3.1. Finally, the front and rear $\mathrm{Ag}$ 
contacts are deposited on unpatterned areas by combining optical lithography and vacuum metal deposition. As illustrated on Figure 7, an unpatterned area located between the front contact and the "photonized" area is left to prevent the metal from contacting the $\mathrm{ZnO}$ layer, which could then shortcircuit the cell.

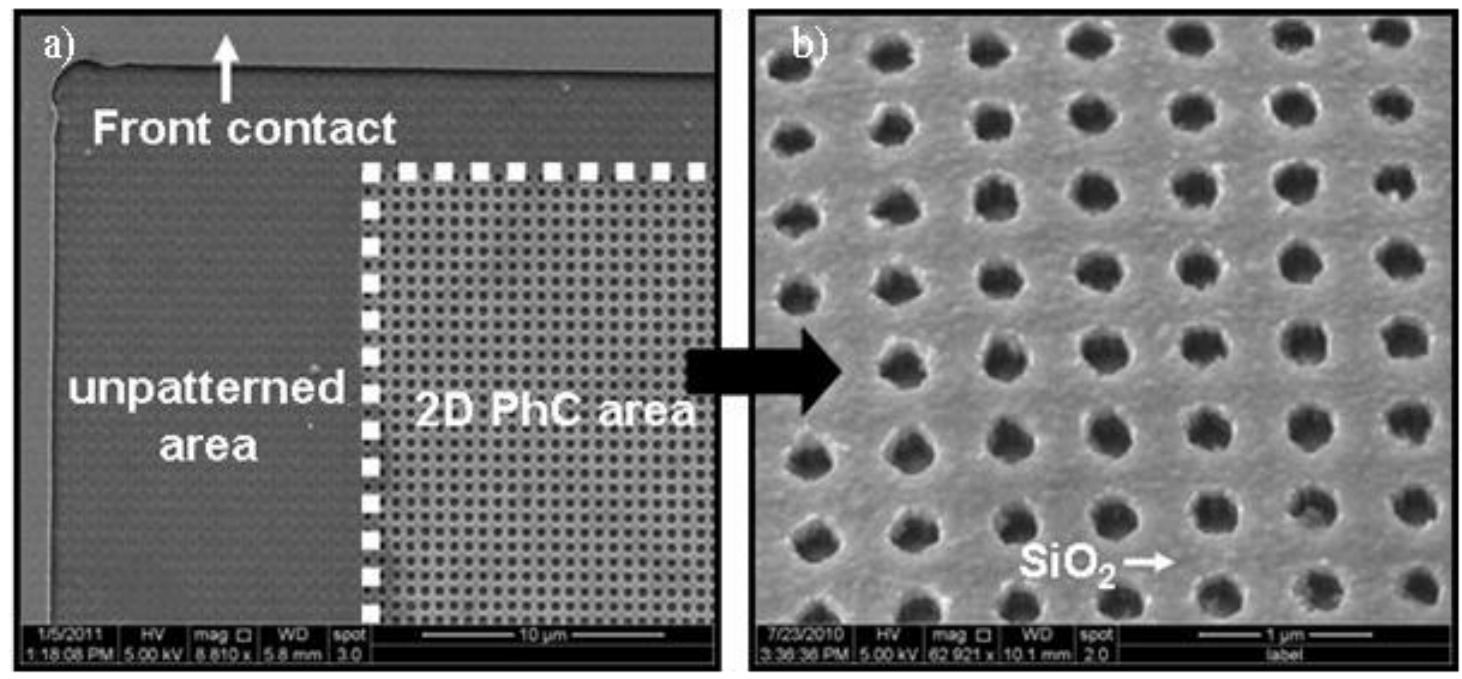

Figure 7. SEM images of a 2D PhC-patterned cell at the end of its fabrication process in the vicinity of the front contacts (a) and on the $\mathrm{PhC}$ region ( $45^{\circ}$ tilted view) (b)

All the aforementioned fabrication steps were validated and several 2D-patterned solar cells have been fabricated so far using this process. Further details can be found in [22].

Another point which needs to be stressed here is the influence of the free surfaces created during the etching which are likely to present higher defect densities. This effect, which will be investigated experimentally in the near future, can already be assessed thanks to electro-optical simulations developed in the next section.

\subsection{Electro-optical characteristics}

So as to achieve the most suited design for our PhC-patterned solar cells, it is important to know to what extent their electrical characteristics are governed by surface recombination. Indeed, one may expect that the surface recombination velocity (SRV) has a greater impact in this case than for the unpatterned cells, even if a final passivation step is planned, thanks to a few nm thick $\left(\mathrm{Al}_{2} \mathrm{O}_{3}, \mathrm{SiN}_{\mathrm{x}}\right)$ layer deposition.

Using the SILVACO electrical simulator enables to compute the short-circuit current density $\left(J_{s c}\right)$ and the open-circuit voltage $\left(V_{o c}\right)$ and thus the yield from the electron-hole generation rate, taking into account the bulk and surface material parameters.

Since the generation rate maps directly derived from the electric field absorption maps, a direct link can be established between optical and electrical simulations. A complete electro-optic optimization of the yield could have even been done. However, it appears that the generation maps present quite similar aspects for various optimized parameters $L$ and $(D / L)$ ratio of the $\mathrm{PhC}$ structure, with the concentration of the electric field close to the interfaces. Thus, electric simulation follows the optical optimization and is aimed at studying the effect of the SRV. 
In order to investigate this point, we performed electro-optical simulations on a planar 1D $\mathrm{PhC}$ structure (with parameters: $L=480 \mathrm{~nm}$ and $D / L=27.5 \%$ ) which should give similar trends as $2 \mathrm{D}$ configurations while being less demanding in terms of computation resources. The carrier generation rate map inside the a-Si:H layer, averaged on both TE and TM polarizations and integrated between $300 \mathrm{~nm}$ and $760 \mathrm{~nm}$ for a light incoming under a normal incidence, was provided by RCWA simulations. At this level, the whole stack presented in the previous sections was considered but only the data related to the active layer were extracted and introduced in the electrical simulator.

For the sake of simplicity, several assumptions were made regarding the a-Si:H properties; in particular, the lifetime of both electron and hole was set to $10^{-7} \mathrm{~s}$, the mobility of the carriers was fixed in doped and intrinsic layers, and a simplified recombination model is used for this study (the recombination at the front and rear surfaces is neglected and band tails and dangling bonds were not modelled). The other parameters used in our simulations can be found in the Appendix.

Firstly, the influence of the SRV on the $I V$ curve of this a-Si:H PIN junction was studied. Figure 8 underlines two different trends [23]: While the short-circuit current is almost not affected by surface recombination, $V_{o c}$ drops rapidly as the SRV increases from 0 to $10^{7} \mathrm{~cm} / \mathrm{s}$.

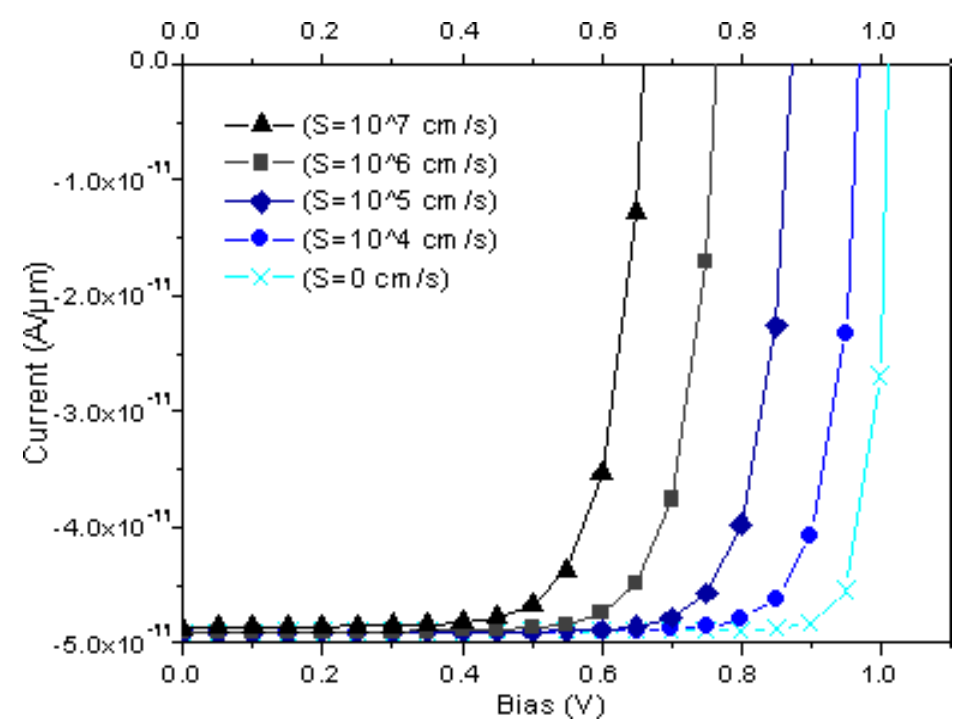

Figure 8. Influence of the surface recombination level $(S)$ on the $I(V)$ characteristics of the optimized 1D-patterned cell

This observation can be explained by considering the distribution of the carrier concentration as well as the electrical field profiles across the junction (see Figures $9 \& 10$ ):

- When $V=0\left(I=I_{s c}\right)$, carriers are mainly concentrated close to the doped regions and benefit from high electrical fields so that they can be efficiently swept away from the intrinsic layer. It can also be noticed that neither the carrier concentration profile nor the electrical field profile are affected by the increase of SRV. For these reasons, carrier recombination is weak, and this is why $I_{s c}$ is less sensitive than $V_{o c}$ to the SRV changes. 


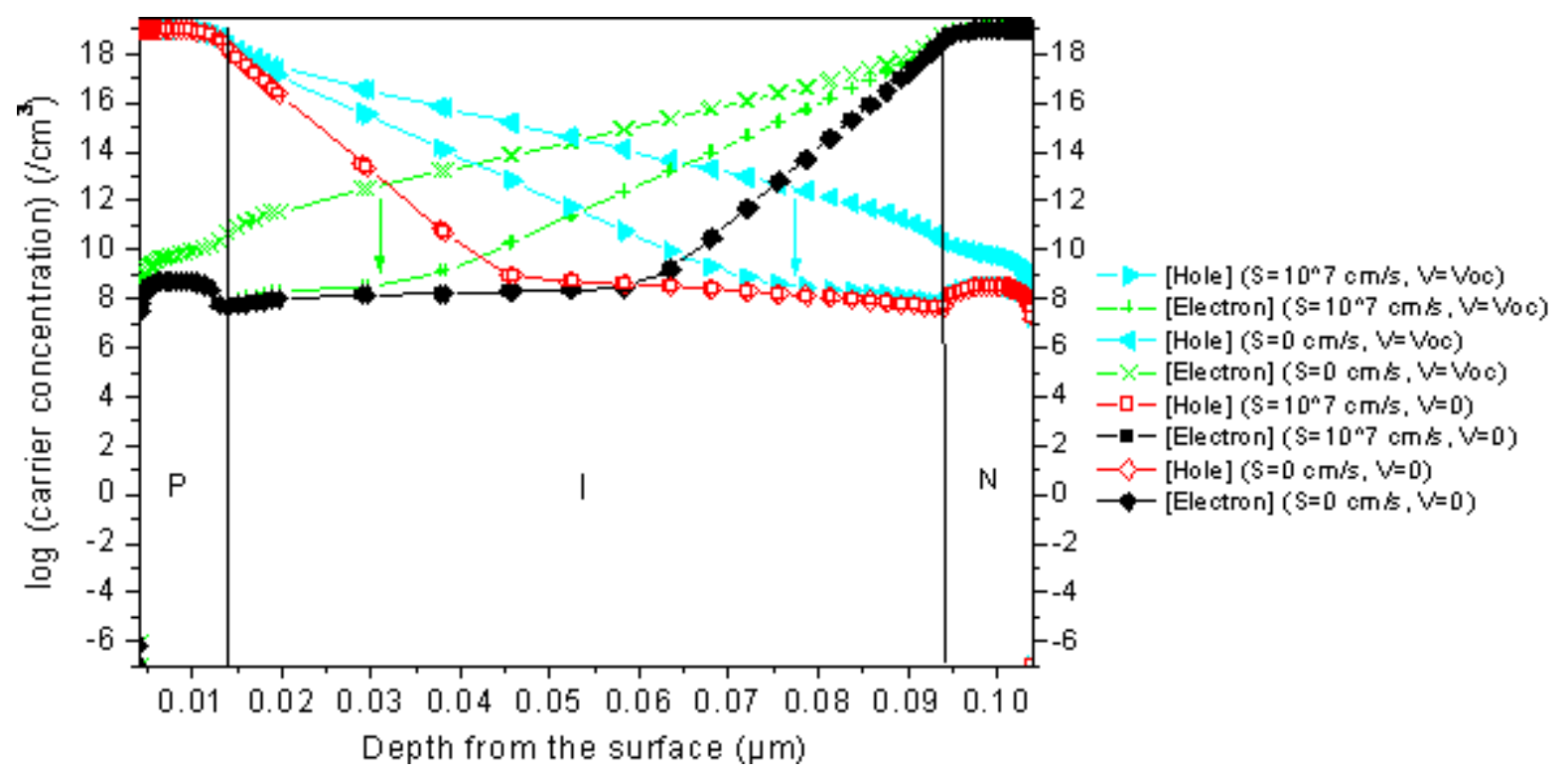

Figure 9. Carrier concentration profiles across the PIN junction of the optimized 1D-patterned cell when $V=0 \mathrm{~V}$ or $V=V_{o c}$ and for two values of the surface recombination $\left(S=0 \mathrm{~cm} / \mathrm{s}\right.$ and $\left.S=10^{7} \mathrm{~cm} / \mathrm{s}\right)$

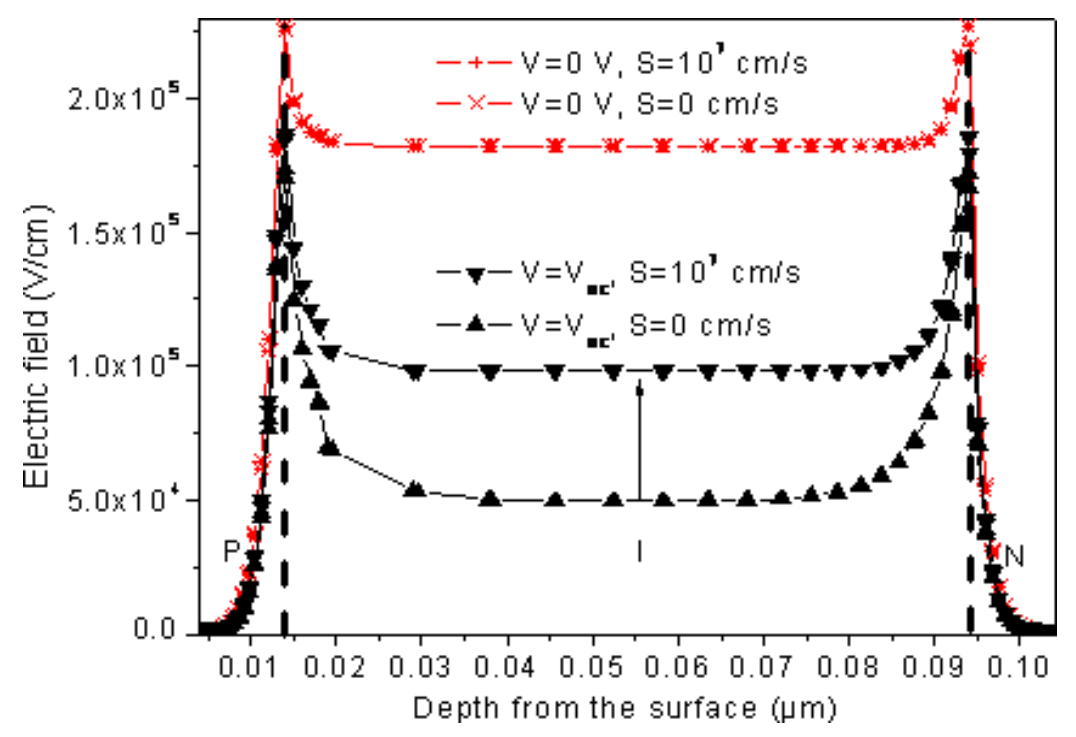

Figure 10. Electric field profiles across the PIN junction of the optimized 1D-patterned cell when $V=0 V$ or $V=V_{o c}$ and for two values of the surface recombination $\left(S=0 \mathrm{~cm} / \mathrm{s}\right.$ and $\left.S=10^{7} \mathrm{~cm} / \mathrm{s}\right)$

- When $V=V_{o c}$, unlike the case where $V=0$, it can be seen that the carriers concentration is important in the intrinsic region. Moreover, the electrical field reaches lower values than for $V=0$, weakening the drifting process of the carriers. This induces that when $V=V_{o c}$, a nonnegligible fraction of the photogenerated carriers can be affected by the surface recombination. Moreover, when the SRV is high $\left(10^{7} \mathrm{~cm} / \mathrm{s}\right.$ on Figure 9), it tends to decrease the carrier concentrations and then to modify the electron and hole quasi-Fermi levels (QFL). Besides, as $V_{o c}$ is proportional to the difference between the QFL of electrons and the QFL of holes, increasing the SRV will reduce the difference of QFLs and therefore, decrease the open-circuit voltage of the cell [24], [25] 
Secondly, we quantified the impact of this $V_{o c}$ (and to a lesser extent, $I_{s c}$ ) decrease on the efficiency of this cell. So as to give a more thorough insight into the surface recombination dependence of the solar cell with regards to its geometrical parameters, we introduced two new configurations having roughly the same integrated absorption in the a-Si:H layer (about 58\%) but with different $\mathrm{CP}$ parameters. More precisely, the first cell $(L=300 \mathrm{~nm}$ and $D / L=45 \%)$ presents more free surfaces than the second one $(L=700 \mathrm{~nm}$ and $D / L=20 \%)$ and should be in principle more sensitive to surface recombination. The results for those three cells are summarized in Figure 11. For comparison, the efficiency $(=8.2 \%)$ of a similar but unpatterned cell is also represented.

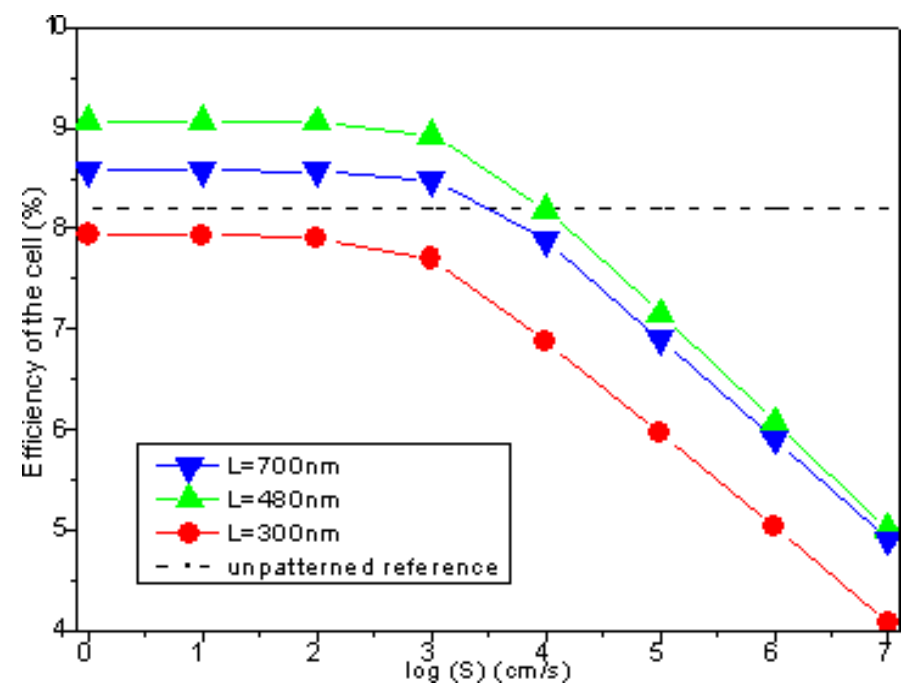

Figure 11. Influence of the surface recombination level $(S)$ on the efficiency of three different 1Dpatterned cells ( $L=300 \mathrm{~nm}, L=480 \mathrm{~nm}$ and $L=700 \mathrm{~nm}$ ) compared to the one of an unpatterned cell (reference)

Without any surface recombination $(S=0 \mathrm{~cm} / \mathrm{s})$, a difference of $0.6 \%$ in efficiency between the $L=300 \mathrm{~nm}$ and $L=700 \mathrm{~nm}$ configurations is already noticeable, although they benefit from the same integrated absorption in the active layer. This can be explained by looking at their generation rate maps displayed in Figure 12. 


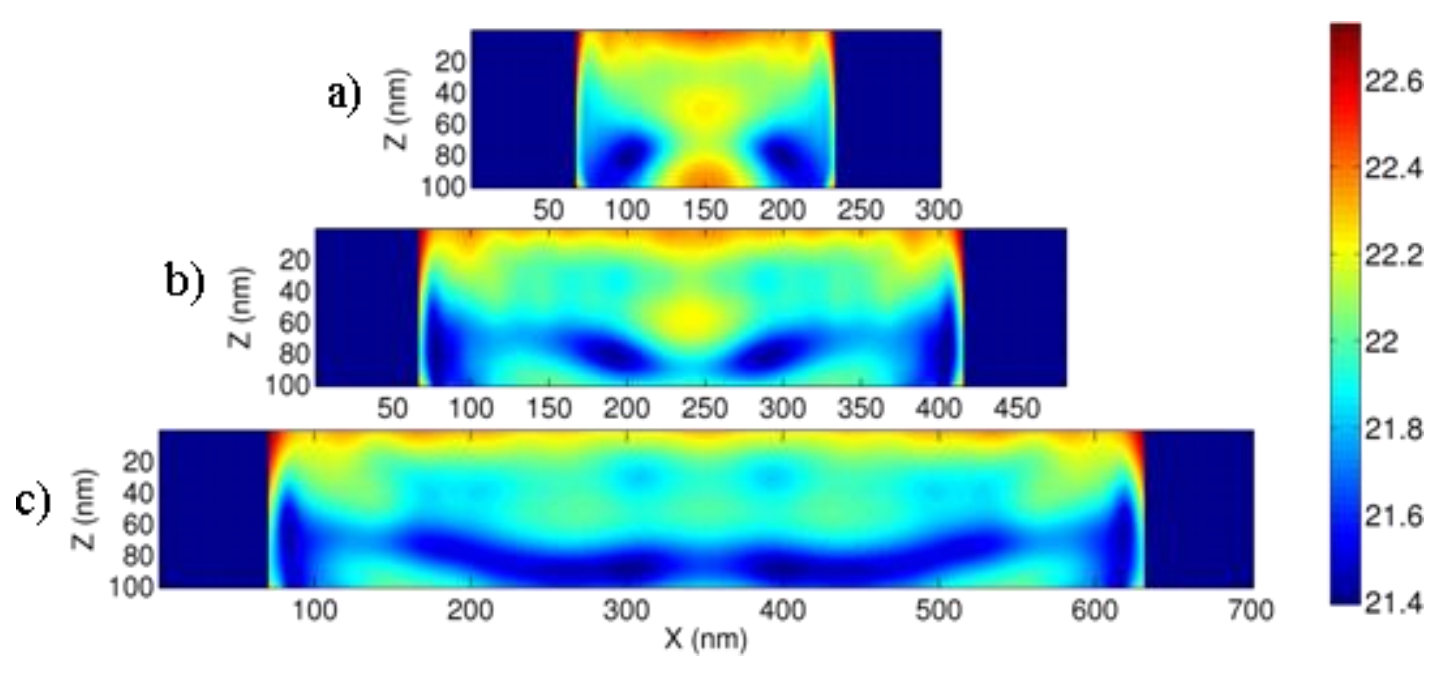

Figure 12. Generation rate maps shown in cross-section for one unit cell of the PIN junction and the ( $L=300 \mathrm{~nm}$ and $D / L=45 \%)$ (a), ( $L=480 \mathrm{~nm}$ and $D / L=27.5 \%)$ (b) and the ( $L=700 \mathrm{~nm}$ and $D / L=20 \%)$ (c) configurations (logarithmic colour scale)

For the $L=300 \mathrm{~nm}$ configuration, many carriers are generated at the front and rear surfaces, in the doped regions, and are then lost because of the high bulk recombination taking place in those layers. For the $L=700 \mathrm{~nm}$ configuration, photogenerated carriers are mainly lost at the front surface only, and this why its efficiency is higher for a similar integrated absorption. Finally, for the $L=480 \mathrm{~nm}$ optimized configuration (integrated absorption in a-Si:H=61.6\%), a part of the carriers is lost in the $\mathrm{p}$ doped region (See Figure 12) but a subsequent fraction of those carriers is also created in the middle of the intrinsic layer, explaining why its efficiency is the highest. More generally, it can be pointed out that the absorption gain obtained by the PhC-patterning cannot be translated into an equivalent efficiency gain due to the photogeneration of carriers in the doped-regions.

When increasing the surface recombination value, the efficiencies of the cells are all dropping in a quasi-similar manner. This indicates that only the carriers located in the direct vicinity of the etched sidewalls are affected by the surface recombination. This could be accounted for by the high electric field involved in such thin junctions, which makes the drift of the carriers particularly efficient. Lastly, it can be seen that the efficiency of the optimized cell $(L=480 \mathrm{~nm})$ reaches the one of the reference for surface recombination as high as $10^{4} \mathrm{~cm} / \mathrm{s}$. This would mean that the etching step should not be too restrictive and when combined with a passivation step, should finally lead to an efficiency gain for the patterned cells.

\subsection{Optical characteristics}

The optical properties of our 2D-patterned cells were measured using an integrating sphere (IS). In this experiment, an unpolarized light was focused at the surface of our cell with an $8^{\circ}$ angle of incidence. The spectrum of the light reflected from the sample at all angles, namely $R(\lambda)$, is then collected. As the stack is deposited on a $\mathrm{Ag}$ back reflector, the absorption spectrum is then simply derived by $A(\lambda) \approx 1$ $R(\lambda)$.

Figure 13 shows the measured and simulated absorption spectrum of a patterned stack with $L=585 \mathrm{~nm}$ and $D / L=47 \%$, together with to the one of a similar but unpatterned stack, which is used as a reference. The experimental spectra are quite comparable to the simulations, including the low wavelength zone, where the PhC structure behaves as an AR structure, and a high wavelength range where additional 
absorption peaks originating from resonant Bloch modes counterbalance the low extinction coefficient of a-Si:H. The discrepancies between simulated and measured spectra are ascribed to some uncertainty regarding the thickness of the layers as well as their optical indices.

In addition, the influence of the angle of incidence is also highlighted by increasing its value from $0^{\circ}$ (simulation) to $16^{\circ}$ (measurements). As can be seen, this leads to a blue-shift the absorption peaks, but it globally leaves the spectrum of the patterned stack unchanged. This can be explained by the multimode nature of the PhC structure which attenuates the effect of a modification in the resonant mode properties.

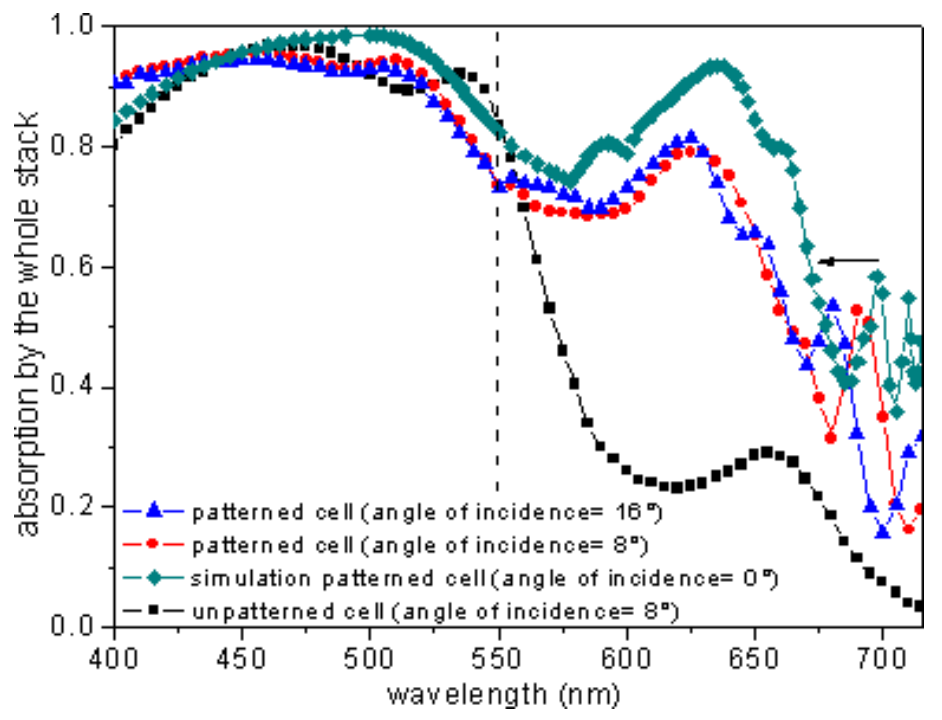

Figure 13. Measured absorption spectra of an unpatterned and a 2D PhC-patterned stack for two angles of incidence. Simulated absorption spectrum of the patterned stack at normal incidence also reported

In order to assess the quality of the PhC structure fabricated by the processes presented in section 3.2, another $\mathrm{PhC}$ patterned solar cell stack $(L=600 \mathrm{~nm}$ and $D / L=58 \%)$ was characterized by microreflectivity $(\mu-\mathrm{R})$, the incident light scanning different areas of around $100 \mu \mathrm{m}$ between the metallic contacts. Shown in Figure 14 is $1-R(\lambda)$ corresponding to these measurements with the absorption spectrum of the unpatterned stack measured with the IS. Although absorption spectra cannot be directly derived from these microreflectivity measurements, since the reflected light is not fully collected, those curves give information on the impact of the $\mathrm{PhC}$ parameters inhomogeneity over the whole cell. From these spectra, one can conclude that the optical response is almost the same in the six probed zones which covers an area of few $\mathrm{mm}^{2}$. This underlines the limited dispersion in the $\mathrm{PhC}$ parameters over the cell as could be inferred from SEM images, together with the robustness of its optical characteristics with regards to those defects, in compliance with optical simulation. 
a)
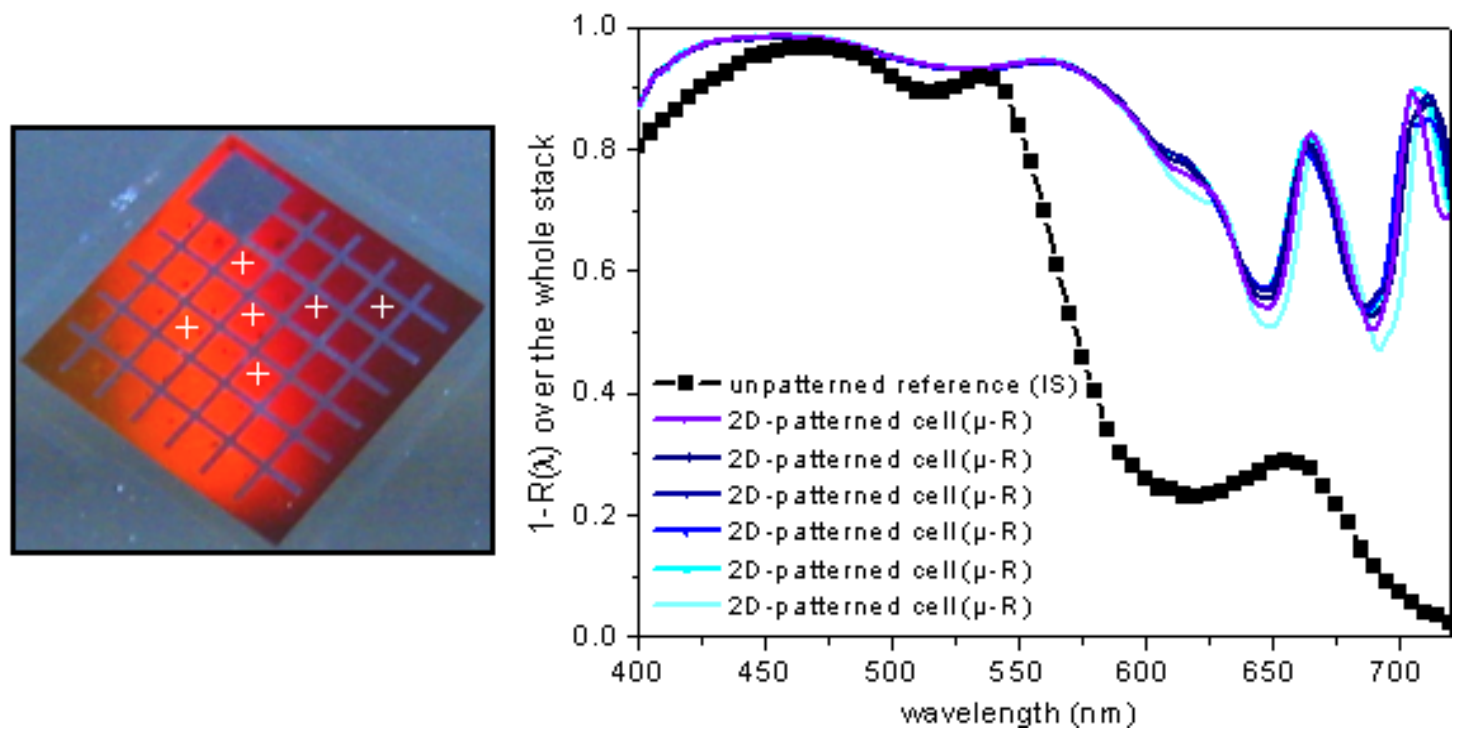

Figure 14. Top-view of a 2D PhC-patterned cell with marks (white crosses) where the measurements were performed (a) and experimental spectra obtained on the different locations of this cell or on an unpatterned reference (b)

\section{Conclusion}

In summary, we introduced with the example of an a-Si:H based thin film solar cell a way to increase its integrated absorption by implementing a 2D PhC structure directly inside the active layer. Thanks to an understanding of the resonances ruling this absorption gain, we proposed a typical design for such a patterned cell, taking into account technological limitations. Optical simulations performed on realistic stacks predict a maximum $40 \%_{\text {rel }}$ increase of integrated absorption in the a-Si:H layer compared to their unpatterned counterparts. By combining holographic lithography with reactive ion etching, 2D patterns were successfully transferred up to the active layer of our cells which were then optically characterized with an integrating sphere and microreflectivity measurements. Experimentally, we demonstrated that the implementation of this 2D-patterning enables an absorption increase over almost the whole spectral range considered. Moreover, this gain is robust with regards to the angle of incidence of the light and the imperfections of the PhC structure, in accordance with simulations. Provided that surface recombination is weakly influencing the electrical characteristics of the cell for reasonable surface recombination velocity values, as suggested by electro-optic simulations, a large part of this absorption gain should be then translated into a global gain of the cell efficiency.

\section{Acknowledgements}

This work was funded by the French Research Agency (ANR) PV program (SPARCS) and supported by the Rhône-Alpes region. PhC patterning and optical characterizations were performed at the NanoLyon Technology Platform on samples fabricated at the Laboratory of Physics of Interfaces and Thin Films (CNRS UMR 7647). Useful discussions with Drs. P. Roca i Cabarrocas and C. Jamois are acknowledged with thanks. The authors express their gratitude to Dr. M. Garrigues for the proofreading of the paper.

\section{Appendix}


Parameters used for the electro-optic simulations in the SILVACO electrical simulator and based on the ATLAS software [26]. It should be noted that those parameters are strongly dependent on the layers deposition conditions and for this reason, we decided to select unfavorable values in the frame of this study:

- PIN junction considered: $10 \mathrm{~nm}$ thick p-doped front layer (doped at $10^{19} \mathrm{~cm}^{-3}$ ), $80 \mathrm{~nm}$ thick intrinsic layer ( $\mathrm{p}$-doped at $10^{14} \mathrm{~cm}^{-3}$ ) and $10 \mathrm{~nm}$ thick n-doped rear layer (doped at $10^{19} \mathrm{~cm}^{-3}$ )

- Effective electron lifetime: $\tau_{\mathrm{n}}=10^{-7} \mathrm{~s}$

- Effective hole lifetime: $\tau_{\mathrm{h}}=10^{-7} \mathrm{~s}$

- Electron mobility: $\mu_{\mathrm{n}}=1 \mathrm{~cm}^{2} \cdot \mathrm{V}^{-1} \cdot \mathrm{s}^{-1}$

- Hole mobility: $\mu_{\mathrm{h}}=10^{-2} \mathrm{~cm}^{2} \cdot \mathrm{V}^{-1} \cdot \mathrm{s}^{-1}$

- Band gap Eg (a-Si:H $)_{300 K}=1.7 \mathrm{eV}$

- Electron affinity (a-Si:H) $\chi=3.85 \mathrm{eV}$

- Dielectric constant $(\mathrm{a}-\mathrm{Si}: \mathrm{H})=11.9$

- Conduction band density of states $(\mathrm{a}-\mathrm{Si}: \mathrm{H})_{300 \mathrm{~K}} \mathrm{~N}_{\mathrm{c}}=2.10^{20} \mathrm{~cm}^{-3}$

- Valence band density of states $(\mathrm{a}-\mathrm{Si}: \mathrm{H})_{300 \mathrm{~K}} \mathrm{~N}_{\mathrm{v}}=2.10^{20} \mathrm{~cm}^{-3}$

- Shockley-Read-Hall (SRH) model used for bulk recombination

\section{References}

[1] Atwater H A and Polman A 2010 Nature Materials 9205

[2] Pillai S, Catchpole K R, Trupke T and Green M A 2007 J. Appl. Phys. 9093105

[3] Pala R A, White J, Barnard E, Liu J and Brongersma M L 2009 Adv. Mat. 213504

[4] Zeng L, Yi Y, Hong C, Liu J, Feng N, Duan X, Kimerling L C and Alamariu B A 2006 Appl. Phys. Lett. 89111111

[5] Seassal C, Park Y, Fave A, Drouard E, Fourmond E, Kaminski A, Lemiti M, Letartre X and Viktorovitch P 2008 Proc.of SPIE 7002700207

[6] Zanotto S, Liscidini M and Andreani L C 2010 Opt. Express. 18 4260-4274

[7] Gomard G, Drouard E, Letartre X, Meng X, Kaminski A, Fave A, Lemiti M, Garcia-Caurel E and Seassal C 2010 J. Appl. Phys. 108123102

[8] Bielawny A, Upping J and Wehrspohn R B 2009 Sol. Energ. Mat. Sol. Cells 93 1909-1912

[9] Zhu J, Yu Z, Burkhard G F, Hsu C M, Connor S T, Xu Y, Wang Q, McGehee M, Fan S and Cui Y 2009 Nano Lett 9 279-282

[10] Ferry V E, Verschuuren M A, Li H B T, Schropp R E I, Atwater H A and Polman A 2009 Appl. Phys. Lett. 95183503

[11] Song Y M, Yu J S and Lee Y T 2010 Optics Lett. 35 276-278

[12] Krc J, Zeman M, Smole F and Topic M 2002 J. Appl. Phys. 92 749-755

[13] Moharam M G and Gaylord T K 1981 J. Opt. Soc. Am. 71 811-818

[14] http://camfr.sourceforge.net/

[15] Seassal C, Monat C, Mouette J, Touraille E, Ben Bakir B, Hattori H, Leclercq J L, Letartre X, Rojo-Romeo P and Viktorovitch P 2005 IEEE Journal on Selected Topics in Quantum Electronics 11 395-407

[16] Mandelshtam V A and Taylor H S 1998 J. Chem. Phys. 107 6756-6769

[17] Sakoda K 2004 Optical Properties of Photonic Crystals (2nd edition) Springer

[18] Park Y, Drouard E, El Daif O, Letartre X, Viktorovitch P, Fave A, Kaminski A, Lemiti M and Seassal C 2009 Opt. Express. 17 14312-14321

[19] Poissant Y, Chatterjee P and Roca I Cabarrocas P 2003 J. Appl. Phys. 947305

[20] Viktorovitch P, Ben Bakir B, Boutami S, Leclercq J L, Letartre X, Rojo-Romeo P, Seassal C, Zussy M, Di Cioccio L and Fedeli J M 2010 Laser \& Photonics Reviews 4401 
[21] Ellmer K, Klein A and Rech B 2008 Transparent Conductive Zinc Oxide: Basics and Applications in Thin Film Solar Cells Heidelberg Springer

[22] Meng X, Gomard G, El Daif O, Drouard E, Orobtchouk R, Kaminski A, Fave A, Lemiti M, Abramov A, Roca i Cabarrocas P and Seassal C 2011 Sol. Energ. Mat. Sol. Cells 95 S32-S38

[23] Gomard G, El Daif O, Drouard E, Meng X, Kaminski A, Fave A, Lemiti M, Garcia-Caurel E, Roca i Cabarrocas P and Seassal C 2010 Proc.of Photonics Europe 11-15 April 2010 Bruxelles 7725 77250M-1 - 77250M-6

[24] Schiff E A 2003 Sol. Energ. Mat. Sol. Cells 78 567-595

[25] Dhariwal S R and Smirty M 2006 Sol.Energ. Mat Sol.Cells 90 1254-1272

[26] SILVACO International, 4701 Patrick Henry Drive, Bldg. 1, Santa Clara, CA 94054 